\title{
An Efficient Coin Recognition System Based on Correlation
}

\author{
Huang Dan ${ }^{1}$ Yang Zheng-rong ${ }^{2}$ Yu Ji-yang ${ }^{3}$ \\ ${ }^{1}$ Science Research Institute of China North Industries Group Corporation \\ ${ }^{2}$ Baotou Iron and Steel Vocational Technical College \\ ${ }^{3}$ China Academy of Space Technology, email: yjywarcraft@163.com
}

\begin{abstract}
An efficient coin recognition system based on correlation is proposed. The system is built by a camera and a computer with the recognition algorithm implemented by software MATLAB. The correlation process in the twodimensional frequency domain utilizes the two-dimensional Fast Fourier Transform to improve the processing speed. The search method is optimized to reduce the number of matching. The proposed system produces the type of the coin, and its rotating angle relative to the reference image. Compared with previous coin recognition system, this proposed is simple and clear, without profound mathematical derivation and highly complex hardware design.
\end{abstract}

Keywords: correlation; coin recognition; MATLAB; camera

\section{Introduction}

Coin recognition system can be seen everywhere, such as the bank's currency of account monitoring system ${ }^{[1]}$. However, the more accurate the more complex the system to be designed will be $\mathrm{e}^{[2][3]}$.

Seth used vector quantization and the statistical distribution model to identify individual coins ${ }^{[6]}$. Fukumi gave a neural network algorithm by the surface characteristics of the extraction of coins in order to identify coin images, and 500
Yen to 500 Won coins were experimented to verify the effectiveness of the algorithm ${ }^{[4][7]}$. Bremananth used matching algorithms, Gabor filtering and backward prediction method to determine the number of coins, and the detection accuracy can reach $92 \%{ }^{[5]}$. Abdolah extracted features of image edges, by the way of rotating decomposition method, to determine the currency ${ }^{[3]}$. These methods avoid the differences caused by the rotation angle.

This paper presents an efficient coin recognition system based on a personal computer; just a normal camera is equipped. The algorithm can be realized with the MATLAB program. Compared to the previous literatures, the proposed system has a easy way to be implemented. The results of process obtained is not only able to determine the types of coins, but also able to determine the rotation angle.

\section{The composition of coin recognition system}

The coin identification system should be a model for complex image processing. The whole system should have all the characteristics of image processing, such as sensors, memory, processor, output display, communications transmission equipment and so on. This section will describe the design of the system's algorithm. 


\subsection{Algorithm description}

Image segmentation is used for many coin recognition methods. The quality of segmentation based on specific needs and requirements of the occasion to measure. The algorithms, only includes the feature extraction and image matching.

Edge detection image processing functions, as commonly used in many professional programmers have a special software modules. In the MATLAB program, the function 'edge' is used to implement this feature. The function 'edge' takes intensity or a binary image I as its input, and returns a binary image BW of the same size as I, with 1's where the function finds edges in $I$ and 0 's elsewhere.

Correlation matching matches point features by matching the full image area in the first image and searching for a highly similar point in the second image. The similarity of each point can be measured using various correlations. The specific steps of the algorithm are as follows:

I. Establish a database system including image of all coins. With $N$ kinds of coins, the set of coin edge extraction images are denoted as $A_{i}=\left\{A_{1}, A_{2}, \ldots, A_{N-1}\right\}, i=$ $1, \ldots, N$. Assuming the coin edge extraction image has $M$ types of angles. The set of the $A_{\mathrm{i}}$-th kind of coin' s edge extraction image are $A_{(i, j)}=\left\{A_{(i, 1)}, A_{(i,}\right.$ 2) $\left., \cdots, A_{(i, M)}\right\}, j=1,2, \cdots, M$. So there are $M N$ types of images.

II. Assuming the collected image data by the camera are denoted as $X$, after edge extracted the data are denoted as $Y$. Then produce a result of the cross correlation between $Y$ and $A_{(i, j)}$ to get $Z_{(i, j)}$. Assume the maximum value of the data $Z_{(i, j)}$ is $P k_{(i, j)}=\max \left(Z_{(i, j)}\right)$, compare all of the $P k_{(i, j)}$ to get the maximum value max $\left(P k_{(i, j)}\right)$, where $i$ represents the index of the coin value. The process of image autocorrelation is as follows:
$Z_{(i, j)}=\operatorname{ifft} 2\left(\mathrm{fft} 2(X) * \operatorname{conj}\left(\mathrm{fft} 2\left(A_{(i, j)}\right)\right)\right)$

Where conj represents conjugation, and $\mathrm{fft} 2$ represents the Fourier transform.

\subsection{Experiments}

This section gives an experiment for the identification test of RMB coins. First, the six kinds of coins of the RMB images are acquainted to get the reference images to match the tested figures; then, by rotating the image to get different angles of the reference image, in this trial each rotation angle of 1 degree; finally, the coins' value are determined by matching the test figure with all the reference images.

Reference images acquisition are through the camera equipment of various types of coins. The length and width of the image should be equal to facilitate the image rotation with any angle. A collection of images for a set of RMB coins are shown in Fig. 1.
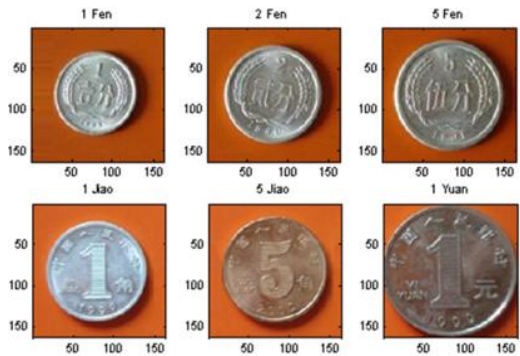

Fig. 1: A set of RMB coins
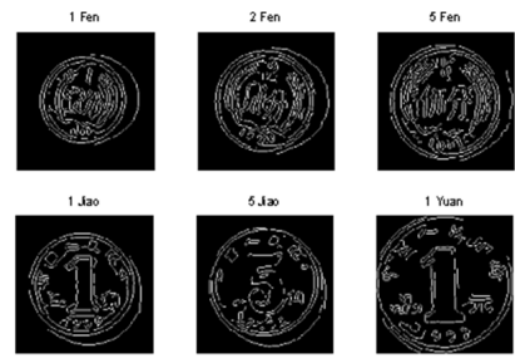

Fig. 2: A set of edge detection figures for RMB Coins

As described in last section, the reference images should go through the edge detection step to reduce the memory occupation. Fig. 2 shows the coin features 
after the image's edge detection processing, with the threshold value of 0.2 . The specific edge detection algorithm can be found in the literature ${ }^{[8]}$. Fig. 3 shows figure examples with a rotate angle of the reference image.
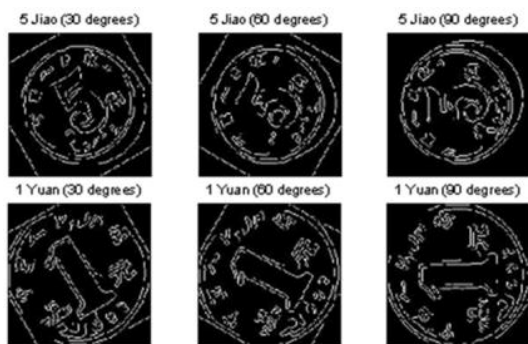

Fig. 3: Figure examples with a rotate angle of the reference image

The 1 Jiao coin in Fig. 4 with counter clockwise rotation angle of 111.8 degrees is tested. The results are shown in Fig. 5. The matching data with a counterclockwise rotation angle of 111.8 degrees and the value 1 Jiao matches the maximum; the results show the correctness of the algorithm. Fig. 6 shows the one dimensional image matching results of test image with the 360 figures of 1 Jiao coin.

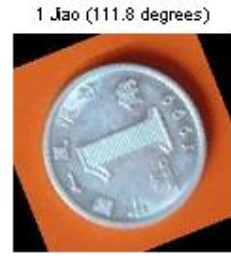

Fig. 4: The coin figure to be detected

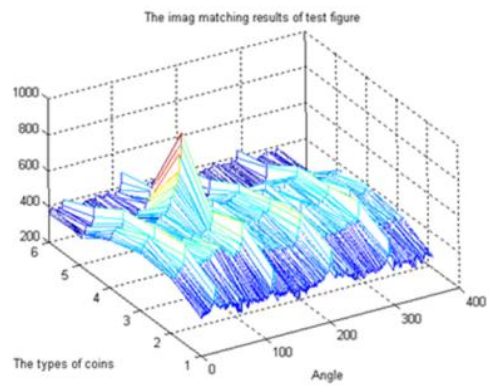

Fig. 5: The image matching results of test figure

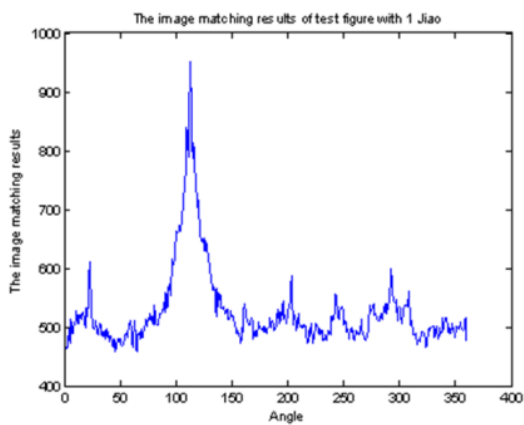

Fig. 6: The image matching results of 1 Jiao

\section{The optimization of coin recogniz- ing algorithm}

This section presents a computational algorithm, based solely on improvements to reduce the computing time. If there are $\mathrm{N}$ kinds of coins, each coin has 360 types of possible angles, and then the matching process needs $360 \mathrm{~N}$ times computations. If the image pixels is the $M^{*} M$, then each time an image similarity calculation required is proportional to $3 * \log _{2}\left(M^{2}\right)$, so the whole calculation time $T$ is $1080 * \log _{2}\left(M^{2}\right)$. This is because the calculation time of the fast Fourier Transform is proportional to $\log N$.

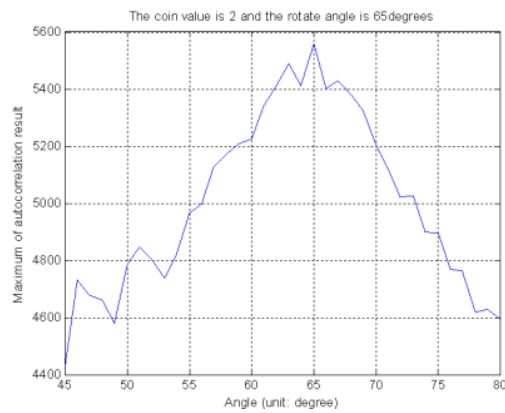

Fig. 7: The matching results of the input coin

This section presents a search algorithm to improve the calculation speed of the image matching process. The results of the matching with same coin is always larger than image matching with different coins, at the same time the same coin of the image matching results are only 
reached the maximum at the same rotate angle, within a few degrees in the maximum rolled around, generally in the range of plus or minus 5 degrees. Fig. 7 shows the matching results of the 2 Fen coin with counterclockwise rotating angle 65 degrees. After attenuation of plus or minus 10 degrees, the maximum gradually reaches stable.

First, a few reference images are selected for each coin type match. The average of the similarity values is deduced for comparison. If an input coin image only are matched with $q$ reference images, the computing time reduced to $q T / 360$. According to the results of several tests, the value of $\mathrm{q}$ should be selected greater than or equal to 3 . In this case, the computing time is reduced to $T / 120$.

Secondly, according to the value of the coin, a more detailed search is performed. The number of searches decreases from 360 to 36 , and the total calculation time reduced to $T / 1200$ from $T / 120$. The calculation time has been greatly reduced; however, this strategy will lead to misjudgment of the image.

Finally, matching the input coin image with the 10 reference images in the best interval, compare the results of the similarity matching; the rotate angle of input coin image can be identified.

\section{Conclusions}

Recognition of coins has been a hot topic internationally, in this paper accurate identification of coins were studied. An efficient coin recognition using an image matching approach was introduced. The proposed method produces two results in the recognition process: the type of the coin, and its rotating angle relative to the reference image. Compared with other algorithms, this algorithm is simple and clear, without profound mathematical derivation and highly complex hardware design.

\section{References}

[1] H. R. Al-Zoubi , "Efficient Coin recognition using a statistical approach," IEEE International Conference on Electro/Information Technology, pp. 1-5, 2010.

[2] C.-M. Chen, S.-Q. Zhang, Y.-F. Chen, "A coin recognition system with rotation invariance," International Conf. on Machine Vision and HumanMachine Interface, pp. 755-757, 2010.

[3] A. Chalechale, "Coin recognition using image abstraction and spiral decomposition," 9th International Symposium on Signal Processing and Its Applications, pp. 1-4, 2007.

[4] M. Fukumi, "Rotation-invariant neural pattern recognition system estimating a rotation angle," IEEE Trans. on Neural Networks, 1997, Vol. 8(3), pp. 568-581.

[5] B. Bremananth, M. Sankari, A. Chitra, "A new approach to coin recognition using neural pattern analysis," IEEE Annual INDICON, pp. 366-370, 2005.

[6] S. McNeill, J. Schipper, T. Sellers, M. C. Nechyba, "Coin recognition using vector quantization and histogram modeling," Florida Conf. on the Recent Advances in Robotics, 2004.

[7] M. Fukumi, "Rotation-invariant neural pattern recognition system with application to coin recognition," IEEE Tran. on Neural Networks, 1992, Vol. 3(2), pp. 272-279.

[8] J. R. Parker, "Algorithms for image processing and computer vision," New York, John Wiley \& Sons, Inc., pp. 23-29, 1997. 